\title{
Earth Currents Near a Monopole Antenna With Symmetrical Top Loading
}

James R. Wait

\begin{abstract}
Expressions for the fields are developed for a vertical ground-based monopole with a cone or disk located at the top of the antenna to simulate umbrella top loading. The current distribution on the structure is assumed. Using spherical-wave functions, the magnetic-field distribution on the ground plane near the base of the antenna is computed and illustrated by graphs. For the case where the antenna is electrically small, the currents flowing on the cone or disk are shown to contribute only slightly to the total field.
\end{abstract}

\section{Introduction}

Transmitting antennas at low radiofrequencies usually take the form of a vertical currentcarrying wire loaded by extensive overhead wires which sometimes take the shape of an umbrella. At the risk of oversimplification, a model for field calculations might be a vertical monopole with the umbrella being represented by a continuous cone. In other words, any asymmetry about the vertical axis is neglected. It is the purpose of the present note to investigate the expected influence of the umbrella or cone loading on the fields produced by the antenna with an assumed current distribution.

\section{List of Principal Symbols}

$r_{1}, \theta_{1}$, and $\phi_{1}=$ spherical coordinates of a variable point on the conical sheet.

$a=$ distance from the apex to the rim of the cone.

$I_{0}=$ current at the base of the vertical member.

$F(z)=$ current distribution along the vertical member.

$h=$ height of the antenna or length of the vertical member.

$\Pi_{z}^{v}=z$ component of the Hertz vector of the currents on the vertical member.

$K=$ the slope of the current distribution on the vertical member (assumed constant).

$f\left(r_{1}\right)=$ the current distribution along a generator of the cone.

$A_{q}=$ coefficient for the power series expansion of $f\left(r_{1}\right)$.

$z^{\prime}=$ a variable point on the vertical member (the integration variable).

$\rho, \phi, z=$ cylindrical coordinates of the observation point $P$.

$H_{t}^{v}=$ tangential magnetic field on the ground plane resulting from the currents on the vertical member.

$\Pi_{\rho}^{c}$ and $\Pi_{z}^{c}=$ the $\rho$ and $z$ components of the Hertz vector of the currents on the cone.

$R=$ distance from the variable point $Q$ on the cone to the observation point $P$.

$r, \theta, z=$ spherical coordinates of the point $P$.

$P_{n}^{m}(\cos \theta)=$ associated Legendre polynomial of argument $\cos \theta$ and orders $n$ and $m$.

$j n(k r)=$ spherical Bessel function of the first type of argument $k r$ and argument $n$.

$h_{n}^{(2)}(k r)=$ spherical Hankel function of the second kind of argument $k r$ and order $n$.

$T_{n}\left(r_{1}, r\right)=j n\left(k r_{1}\right) h_{n}^{(2)}(k r)$.

$H_{t}^{d}=$ tangential magnetic field on the ground plane resulting from the currents on the disk or the cone of angle $\theta_{1}=90^{\circ}$.

$A_{n}\left(r_{0}\right), B_{n}\left(r_{0}\right), C_{n}\left(r_{0}\right)=$ coefficients for the spherical-harmonic expansion of $H_{t}^{d}$.

$r_{0}$ and $\theta_{0}=$ spherical coordinates of a variable point on the ground plane for the origin at $O$ in figure $1, r_{0}=\left(\rho^{2}+h^{2}\right)$ and $\theta_{0}=\arctan (\rho / h)$. 
$j^{v}=-a H_{t}^{v} / I_{0} \ldots=$ earth current (dimensionless) on the ground plane resulting from the currents on the vertical member.

$j^{d}=-a H_{t}^{d} / I_{0} \ldots=$ earth current (dimensionless) on the ground plane resulting from the currents on the disk.

$\bar{r}, \bar{\theta}=$ new spherical coordinates for describing the radiation pattern (see fig. 3).

$J_{0}(S), J_{1}(S)$, and $J_{2}(S)$ are cylindrical Bessel functions of the first type of orders 1,2 , and 3 of the argument $S$.

\section{Some Preliminary Considerations}

The top-loaded antenna in its idealized form is shown in figure 1. It consists of a vertical current-carrying wire extending from the point $O$, at $z=0$, along the axis of a cylindrical coordinate system to the ground surface at $z=h$. The point $O$ is also the apex of a cone which is generated by a line of length, $a$, at an angle $\theta_{1}$ to the $z$ axis. With respect to a spherical coordinate system with the origin at $O$, the coordinates of the bottom rim of the cone are $(a$, $\left.\theta_{1}, \phi\right)$ and those of a variable point $Q$ on the cone are $\left(r_{1}, \theta_{1}, \phi_{1}\right)$. The upward current on the central wire filament is taken to be $I_{0} F(z)$ where $I_{0}$ is the current at the apex, i.e., $F(0)=1$, and $I_{0} F(h)$ is the current at the base. The outward current on an element $d r_{1} d \phi_{1}$ on the conical surface is then $\left(I_{0} / 2 \pi\right) d \phi_{1} f\left(r_{1}\right)$ where $f(0)=1$ and $f(a)=0$. Because of symmetry the current on the conical sheet has only a component in the direction of increasing $r_{1}$.

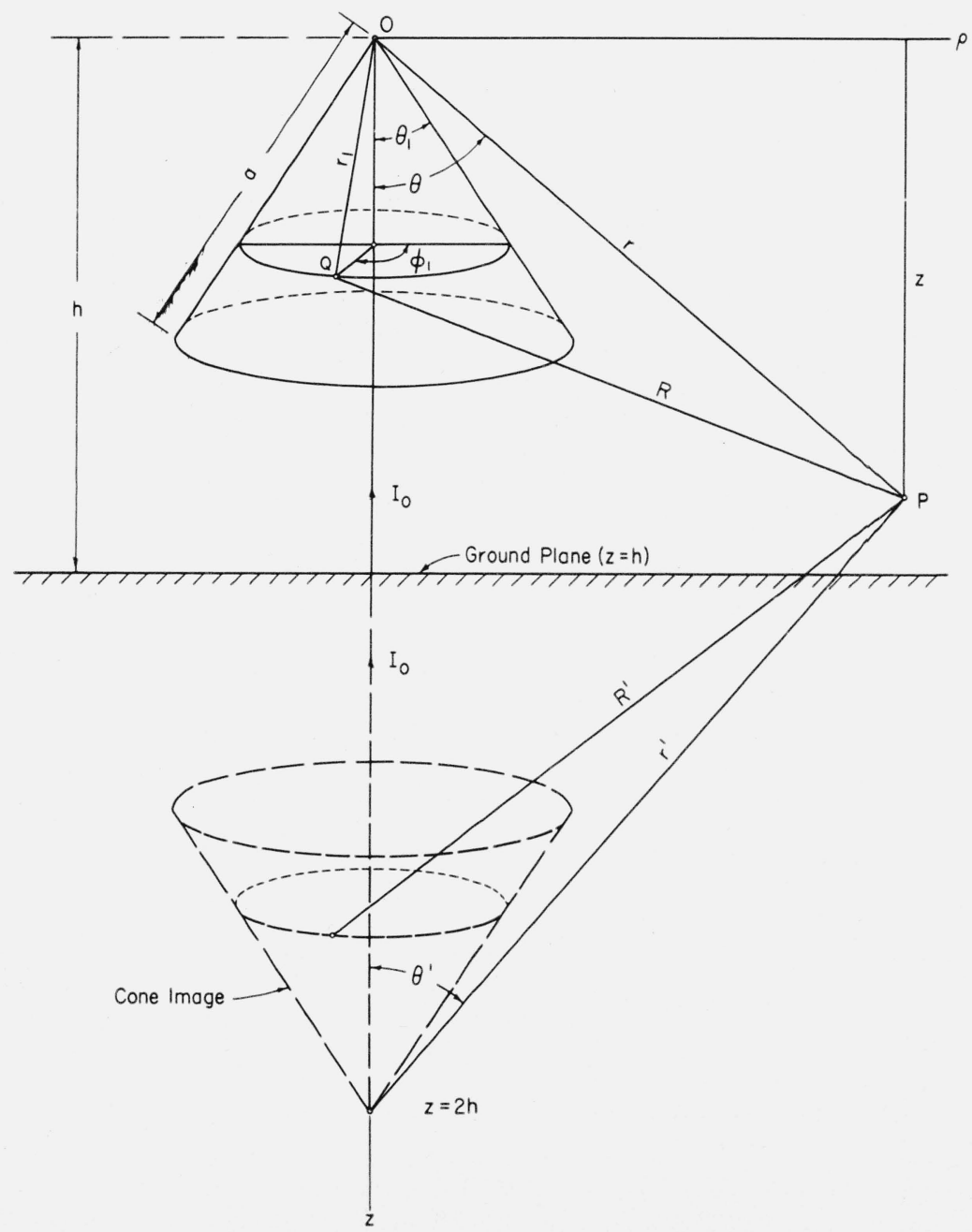

Figure 1. Coordinate system for monopole with cone lcading. 
The current distributions, characterized by the functions $F(z)$ and $f\left(r_{1}\right)$, in general, have a very complicated dependence on the antenna dimensions. In the absence of a top loading and where the vertical member can be approximated by a circular cylinder, current distributions can be expressed in terms of an expansion parameter which is proportional to $\log (h / 2 \pi c)$ where $c$ is the radius of the conductor [1]. ${ }^{1}$ When $h$ is less than one-half wavelength, the distribution is very close to being sinusoidal so long as $h$ is greater than about $100 \mathrm{c}$. In fact, at very low frequencies where $h$ becomes small compared with the wavelength, the current is then almost a linear function of height. It would not be expected that the cone would modify this conclusion. Therefore

$$
F(z) \approx I_{0}(1-K z / h)
$$

where $K$ is a constant which depends on the extent of the top loading. For example, in the unloaded case, $K=1$, and in the ideally loaded case, $K=O$, corresponding to a constant current on the vertical member. In most applications where top loading is employed it is known on experimental grounds [3] that $K \approx O$. This is particularly so for high-power installations at low radiofrequencies where the effective dimension of $a$ is comparable to or greater than $h$, and $\theta_{1}$ is near $90^{\circ}$.

Information regarding the current distribution function $f\left(r_{1}\right)$ for the conical sheet is apparently not available in the literature. However, if each individual wire making up the cone acts independently, it could be expected that the total current flowing towards the rim of the cone would vary in a linear manner with $r$, if $a$ is small compared to the wavelength. On the other hand, when the cone degenerates into a disk (i.e., $\theta_{1} \approx 90^{\circ}$ ) and, if $h$ is somewhat smaller than $a$, it can be expected the total current flowing towards the rim will vary with the square of $r_{1}$. This conclusion is based on the idea that the shunt displacement current density between the circular disk and the ground is a constant if fringing effects at the edges are neglected. A plausible assumed distribution would be

$$
f\left(r_{1}\right)=\sum_{q}\left(\frac{r_{1}}{a}\right)^{q} A_{q}
$$

where the summation could be over $q=0$ and 1 and $A_{0}=1, A_{1}=-1$ for the linear current approximation; whereas $q=0$ and 2 with $A_{0}=1$ and $A_{2}=-1$ for the squared current approximation. It would be possible to employ more elaborate forms of $f\left(r_{1}\right)$ by utilizing more than two values of $q$. A prime requirement is always

$$
\sum_{q} A_{q}=0
$$

since $f(a)=0$.

\section{Field of the Vertical Member}

The Hertz vector $\vec{\Pi}^{v}$ at the point $P(\rho, 0, z)$ due to the current flowing only on the vertical member is given by $\vec{\Pi}^{v}=\left(0,0, \Pi_{z}^{v}\right)$, where

$\Pi_{z}^{v}=\frac{I_{0}}{4 \pi i \omega \epsilon_{0}}\left\{\int_{0}^{h} \frac{\exp \left[-i k\left[\left(z-z^{\prime}\right)^{2}+\rho^{2}\right]^{1 / 2}\right]}{\left[\left(z-z^{\prime}\right)^{2}+\rho^{2}\right]^{1 / 2}} F\left(z^{\prime}\right) d z^{\prime}+\int_{0}^{h} \frac{\exp \left[-i k\left[\left(2 h-z^{\prime}-z\right)^{2}+\rho^{2}\right]^{1 / 2}\right]}{\left[\left(2 h-z^{\prime}-z\right)^{2}+\rho^{2}\right]^{1 / 2}} F\left(z^{\prime}\right) d z^{\prime}\right\}$,

when $k=2 \pi /$ wavelength and $\epsilon_{0}=8.854 \times 10^{-12}$. The fields are then given by

$$
E_{\rho}^{v}=\frac{\partial^{2} \Pi_{z}^{v}}{\partial \rho \partial z}, \quad E_{z}^{v}=\left(k^{2}+\frac{\partial^{2}}{\partial z^{2}}\right) \Pi_{z}^{v}, \quad \text { and } \quad H_{\phi}^{v}=-i \epsilon_{0} \omega \frac{\partial \Pi_{z}}{\partial \rho}
$$

\footnotetext{
1 Figures in brackets indicate the literature references at the end of this paper.
} 
The second integral in eq (4) can be identified with the image of the vertical member below the plane $z=h$. It can be verified that $E_{\rho}^{v}=0$ at $z=h$, which is the required boundary condition for the ideally conducting ground.

The tangential magnetic field, $H_{t}$, on the ground plane is then explicitly given by

$$
H_{t}^{v}=-\frac{I_{0}}{2 \pi} \frac{\partial}{\partial \rho} \int_{0}^{h} \frac{F\left(z^{\prime}\right) \exp \left\{-i k\left[\left(z^{\prime}-h\right)^{2}+\rho^{2}\right]^{1 / 2}\right\}}{\left[\left(z^{\prime}-h\right)^{2}+\rho^{2}\right]^{1 / 2}} d z^{\prime} .
$$

Considering the important case where $\beta h<<1$ and $F\left(z^{\prime}\right) \approx 1$, it follows that

$$
\begin{aligned}
H_{t}^{v} & \approx-\frac{e^{-i k_{\rho}} \rho I_{0}}{2 \pi} \int_{0}^{h}\left[\frac{1}{\left[\rho^{2}+\left(z^{\prime}\right)^{2}\right]^{3 / 2}}+\frac{i k}{\rho^{2}+\left(z^{\prime}\right)^{2}}\right] d z^{\prime} \\
& \approx-\frac{e^{-i k_{\rho}} I_{0}}{2 \pi \rho}\left[\frac{h}{\left(\rho^{2}+h^{2}\right)^{1 / 2}}+i k \rho \tan ^{-1}\left(\frac{h}{\rho}\right)\right] .
\end{aligned}
$$

This can be expanded in a power series in $(h / \rho)$, so that

$H_{t}^{v} \approx-\frac{e^{-i k_{\rho}} I_{0}}{2 \pi \rho}\left[\left(\frac{h}{\rho}\right)(1+i k \rho)-\left(\frac{h}{\rho}\right)^{3}\left(\frac{1}{2}+\frac{i}{3} k \rho\right)+\ldots\right.$ terms containing $\left(\frac{h}{\rho}\right)^{5},\left(\frac{h}{\rho}\right)^{7}$, etc. $]$.

The first term of this expansion corresponds to the field of a hertzian dipole radiator. The additional terms can be regarded corrections to account for the finite value of the antenna height and can be called multipole terms. Sufficiently close to the base, such that $k \rho<<1$, the following expression will suffice

$$
H_{t}^{v} \approx-\frac{I_{0}}{2 \pi} \frac{h}{\rho\left(\rho^{2}+h^{2}\right)^{1 / 2}}
$$

which is a quasi-static approximation. A check on this result is obtained by noting that

$$
\lim _{\rho \rightarrow 0} H_{t}^{v}=-\frac{I_{0}}{2 \pi \rho}
$$

which could be predicted from Ampere's law.

\section{Field of the Conical Currents}

From an argument based on symmetry, it can be seen that the Hertz vector $\vec{\Pi}^{c}$ at $P$, due to the currents flowing in the cone, has a vanishing $\phi$ component. The $\rho$ and $z$ components, however, are, in general, nonvanishing. These can be expressed as an integration over the area of the cone, such that,

and

$$
\Pi_{\rho}^{c}=\frac{\left(I_{0} / 2 \pi\right) \sin \theta_{1}}{4 \pi i \epsilon_{0} \omega} \int_{0}^{2 \pi} d \phi_{1} \int_{0}^{a} d r_{1} \cos \phi_{1} \frac{e^{-i k R}}{R} f\left(r_{1}\right),
$$

$$
\Pi_{z}^{c}=\frac{\left(I_{0} / 2 \pi\right) \cos \theta_{1}}{4 \pi i \epsilon_{0} \omega} \int_{0}^{2 \pi} d \phi_{1} \int_{0}^{a} d r_{1} \frac{e^{-i k R}}{R} f\left(r_{1}\right),
$$

where $R^{2}=r^{2}+r_{1}^{2}-2 r r_{1} \cos \Omega$ and $\cos \Omega=\cos \theta \cos \theta_{1}+\sin \theta \sin \theta_{1} \cos \phi_{1}$. Now the factor $1 / R$ is a solution of Laplace's equation and can be written in terms of spherical harmonics, as is well known [2],

$$
\frac{1}{R}=\sum_{n=0}^{\infty} \sum_{m=0}^{n} \epsilon_{m} \frac{(n-m) !}{(n+m) !} P_{n}^{m}(\cos \theta) P_{n}^{m}\left(\cos \theta_{1}\right) \cos m \phi_{1}\left\{\begin{array}{l}
r_{1}^{n} / r^{n+1} ; r>r_{1} \\
r^{n} / r_{1}^{n+1} ; r_{1}>r .
\end{array}\right.
$$

where $\epsilon_{0}=1, \epsilon_{m}=2$ if $(m \neq 0)$, and where the $P_{n}^{m}$ are associated Legendre polynomials. The factor $e^{-i k R} / R$, on the other hand, is a solution of the wave equation and an expansion for it in terms of spherical wave functions can be expected. Such an expansion does not seem to be available; 
it can be obtained, somewhat heuristically, by noting that it should reduce to eq (12) for $\mathrm{kr}$ and $k r_{1}$ small, and should give rise to outgoing wave at infinity. Such an expansion is

$\frac{e^{-i k R}}{R}=-i k \sum_{n=0}^{\infty} \sum_{m=0}^{n} \epsilon_{m} \frac{(n-m) !}{(n+m) !}(2 n+1) P_{n}^{m}(\cos \theta) P_{n}^{m}\left(\cos \theta_{1}\right) \cos m \phi_{1}\left\{\begin{array}{l}j n\left(k r_{1}\right) h_{n}^{(2)}(k r) ; r>r_{1} \\ j n(k r) h_{n}^{(2)}\left(k r_{1}\right) ; r_{1}>r,\end{array}\right.$

where $j n\left(k r_{1}\right)$ is a spherical Bessel function and $h_{n}^{(2)}(k r)$ is a spherical Hankel function of the second kind. These are defined by

and

$$
j n(x)=(-1)^{n} x^{n}\left(\frac{d}{x d x}\right)^{n}\left(\frac{\sin x}{x}\right)
$$

$$
h_{n}^{(2)}(x)=i(-1)^{n}\left(\frac{d}{x d x}\right)^{n}\left(\frac{e^{-i x}}{x}\right) .
$$

The integration, with respect to $\phi_{1}$, in eq (10) and (11) requires the evaluation of

and

$$
I_{1}(r, \theta)=\frac{1}{2 \pi} \int_{0}^{2 \pi} \frac{e^{-i k R}}{R} \cos \phi_{1} d \phi_{1}
$$

$$
I_{2}(r, \theta)=\frac{1}{2 \pi} \int_{0}^{2 \pi} \frac{e^{-i k R}}{R} d \phi_{1}
$$

which can now be rewritten

and

$$
I_{1}(r, \theta)=-i k \sum_{n=1}^{\infty} \frac{(2 n+1)}{n(n+1)} P_{n}^{1}(\cos \theta) P_{n}^{1}\left(\cos \theta_{1}\right)\left\{\begin{array}{l}
T_{n}\left(r_{1}, r\right) ; r>r_{1} \\
T_{n}\left(r, r_{1}\right) ; r_{1}>r
\end{array}\right.
$$

$$
I_{2}(r, \theta)=-i k \sum_{n=0}^{\infty}(2 n+1) P_{n}^{0}(\cos \theta) P_{n}^{0}\left(\cos \theta_{1}\right)\left\{\begin{array}{l}
T_{n}\left(r_{1}, r\right) ; r>r_{1} \\
T_{n}\left(r, r_{1}\right) ; r_{1}>r
\end{array}\right.
$$

where $T_{n}\left(r_{1}, r\right)=j n\left(k r_{1}\right) h_{n}^{(2)}(k r)$.

The circumferential field $H_{\phi}^{c}$ due to the conical currents is given by

$$
\begin{aligned}
& H_{\phi}^{c}=i \epsilon_{0} \omega\left[\frac{\partial \Pi_{p}^{c}}{\partial z}-\frac{\partial \Pi_{z}^{c}}{\partial \rho}\right], \\
& H_{\phi}^{c}=i \epsilon_{0} \omega\left[\frac{\partial \Pi_{p}^{c}}{\partial r} \cos \theta-\frac{\partial \Pi_{p}^{c}}{r \partial \theta} \sin \theta-\frac{\partial \Pi_{z}^{c}}{\partial r} \sin \theta-\frac{\partial \Pi_{z}^{c}}{r \partial \theta} \cos \theta\right],
\end{aligned}
$$

where

$$
i \epsilon_{0} \omega \Pi_{\rho}^{c}=\frac{I_{0} \sin \theta_{1}}{4 \pi} \int_{0}^{a} f\left(r_{1}\right) I_{1}(r, \theta) d r_{1}
$$

and

$$
i \epsilon_{0} \omega \Pi_{z}^{c}=\frac{I_{0} \cos \theta_{1}}{4 \pi} \int_{0}^{a} f\left(r_{1}\right) I_{2}(r, \theta) d r_{1} .
$$

To satisfy the boundary conditions at the ground plane, $z=h$, the fields of the mirror image of the cone must be added to $H_{\phi}^{c}$. For these fields $I_{1}(r, \theta)$ must be replaced by $-I_{1}\left(r^{\prime}, \theta^{\prime}\right)$ and $I_{2}(r, \theta)$ by $+I_{2}\left(r^{\prime}, \theta^{\prime}\right)$ where $\theta^{\prime}=\mathrm{M}-\theta, r^{\prime}=\left[(2 \mathrm{~h}-z)^{2}+\rho^{2}\right]^{\frac{1}{2}}$, and $\cos M=\left[(r)^{2}+\left(r^{\prime}\right)^{2}-4 h^{2}\right] / 2 r r^{\prime}$.

The preceding equations, along with eq (7) and (8), constitute the complete formal solution for the fields of a cone-loaded monopole for a specified current distribution on the structure.

\section{Disk-Loaded Monopole}

When the angle $\theta_{1}$ approaches $90^{\circ}$, the cone degenerates into a disk. It is this case that will be considered in some detail here. Of particular interest is the behavior of the currents excited on the ground plane in the vicinity of the disk. In fact, for vlf antennas these nearzone currents have, associated with them, considerable ohmic losses due to the imperfect conduc- 
tivity of the soil [4]. The surface current density is by definition numerically equal to the tangential magnetic field $H_{t}^{d}$ on the ground plane. For the case $k r<<1$, it follows that

$$
H_{t}^{d}=\frac{I_{0}}{2 \pi} \sum_{n=1}^{\infty}\left[A_{n}\left(r_{0}\right) P_{n+1}^{1}\left(\cos \theta_{0}\right)+B_{n}\left(r_{0}\right) P_{n-1}^{1}\left(\cos \theta_{0}\right)\right] P_{n}^{1}(0) \text { for } r_{0}<a
$$

and

$$
H_{t}^{d}=\frac{I_{0}}{2 \pi} \sum_{n=1}^{\infty} C_{n}\left(r_{0}\right) P_{n+1}^{1}\left(\cos \theta_{0}\right) P_{n}^{1}(0) \text { for } r_{0}>a,
$$

where

$$
\begin{aligned}
& A_{n}\left(r_{0}\right)=-\int_{0}^{r_{0}} \frac{f\left(r_{1}\right) r_{1}^{n}}{(n+1) r_{0}^{n+2}} d r_{1}, \\
& B_{n}\left(r_{0}\right)=\int_{r_{0}}^{a} \frac{f\left(r_{1}\right) r_{0}^{n-1}}{n r_{1}^{n+1}} d r_{1}, \\
& C_{n}\left(r_{0}\right)=-\int_{0}^{a} \frac{f\left(r_{1}\right) r_{1}^{n}}{(n+1) r_{0}^{n+2}} d r_{1},
\end{aligned}
$$

and $r_{0}=\left(\rho^{2}+h^{2}\right)^{1 / 2}$ and $\theta_{0}=\arctan (\rho / h)$.

Setting

$$
f\left(r_{1}\right)=\sum_{q} A_{q}\left(r_{1} / a\right)^{q}
$$

it is a simple matter to arrive at

$$
\begin{aligned}
& A_{n}\left(r_{0}\right)=-\frac{1}{r_{0}} \sum_{q} \frac{\left(r_{0} / a\right)^{q} A_{q}}{(n+1)(n+q+1)}, \\
& B_{n}\left(r_{0}\right)=\sum_{q} \frac{1}{n r_{0}(q-n)}\left[\left(\frac{r_{0}}{a}\right)^{n}-\left(\frac{r_{0}}{a}\right)^{q}\right] A_{q}, \\
& C_{n}\left(r_{0}\right)=-\sum_{q} \frac{1}{r_{0}} \frac{\left(a / r_{0}\right)^{n+1} A_{q}}{(n+q+1)(n+1)} .
\end{aligned}
$$

Setting $A_{0}=1, A_{2}=-1$ and the other $A_{q}{ }^{\prime}$ s equal to zero, the squared current distribution, $f\left(r_{1}\right)=1-\left(r_{1}^{2} / a^{2}\right)$, is attained. Numerical values of the normalized current, $j^{d}=-a H_{t}^{d} / I_{0}$ and $j^{v}=-a H_{t}^{v} / I_{0}$ are shown plotted in figure 2 as a function of $\rho / a$ for the squared current distribution on the disk and a constant current on the vertical member. The values of the ratio $h / a$ are shown on the curves. The resultant current $j$ on the ground plane which is radially directed is given simply by $j^{d}+j^{v}$ in the near-zone region where $\rho$ is small compared to the wavelength.

It is of interest to examine the radiation field such that $r>>$ wavelength. The spherical Hankel functions can then be well approximated by the first term of their asymptotic expansion. The radiation field $H_{\phi}^{d}$ of the disk by itself is then given by

$$
H_{\phi}^{d} \approx-\frac{i k I_{0} e^{-i k r}}{4 \pi r} \cos \theta \sum_{n=1}^{\infty} \frac{(2 n+1)}{n(n+1)} P_{n}^{1}(\cos \theta) P_{n}^{1}(0) \int_{r_{1}=0}^{a} j n\left(k r_{1}\right) f\left(r_{1}\right) d r_{1} .
$$

The radiation of the disk and its image is obtained by adding $H_{\phi}^{d^{\prime}}$ to the preceding equation which is of the same form as $-H_{\phi}^{d}$ with $\pi-\theta$ replacing $\theta$, and $r-2 h k \cos \theta$ replacing $r$. The summation in the preceding can be converted to a Bessel function by first starting with the addition theorem;

$$
\exp \left(i k r_{1} \sin \theta \cos \phi\right)=\sum_{m=0}^{n} \sum_{n=0}^{\infty} \epsilon_{m} \frac{(n-m) !}{(n+m) !}(2 n+1) P_{n}^{m}(\cos \theta) P_{n}^{m}(0) \cos m \phi j n\left(k r_{1}\right),
$$




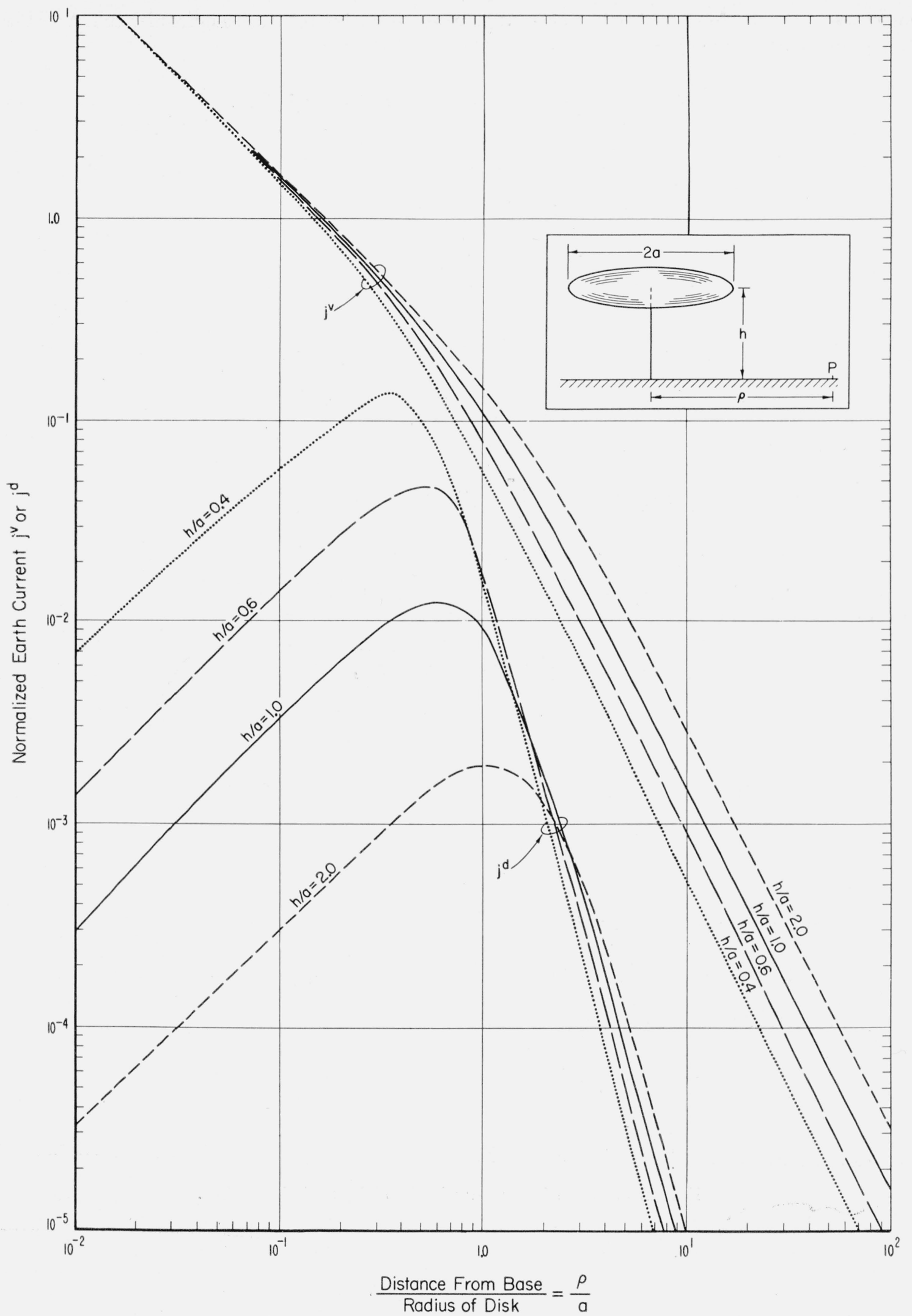

Figure 2. Earth current distribution below a disk-loaded monopole. 
and then multiplying each side by $\cos \phi$ and integrating with respect to $\phi$ from 0 to $2 \pi$. This leads to the interesting relation

$$
i J_{1}\left(k r_{1} \sin \theta\right)=\sum_{n=1}^{\infty} \frac{(2 n+1)}{n(n+1)} P_{n}^{1}(\cos \theta) P_{n}^{1}(0) j n\left(k r_{1}\right) .
$$

Therefore

$$
H_{\phi}^{d} \approx \frac{k I_{0} e^{-i k r}}{4 \pi r} \cos \theta \int_{r_{1}=0}^{a} f\left(r_{1}\right) J_{1}\left(k r_{1} \sin \theta\right) d r
$$

subject, of course, to $k r>>1$. The squared-current assumption, $f\left(r_{1}\right)=1-\left(r_{1}^{2} / a^{2}\right)$ is now introduced in the above which fortunately enables the integration to be carried out to yield

$$
H_{\phi}^{d}=\frac{I_{0} k a e^{-i k r}}{4 \pi r} \cos \theta T(S),
$$

where $T(S)=\left[1-J_{0}(S)-J_{2}(S)\right] S^{-1}$ with $S=k a \sin \theta$. The field of disk plus its image can then be written

$$
H_{\phi}^{d}+H_{\phi}^{d \prime} \approx \frac{I_{0} k a e^{-i k \bar{r}}}{2 \pi \bar{r}} \cos \bar{\theta} i \sin (k h \cos \bar{\theta}) T(k a \sin \bar{\theta})
$$

in terms of the spherical coordinates $\bar{r}, \bar{\theta}$ with the origin at the base of the vertical member as indicated in figure 3. For the usual case where $k a \sin \theta<<1$, it follows from the power series expansions of the Bessel functions $J_{0}$ and $J_{2}$, that

$$
T(k a \sin \bar{\theta}) \approx \frac{k a \sin \bar{\theta}}{8},
$$

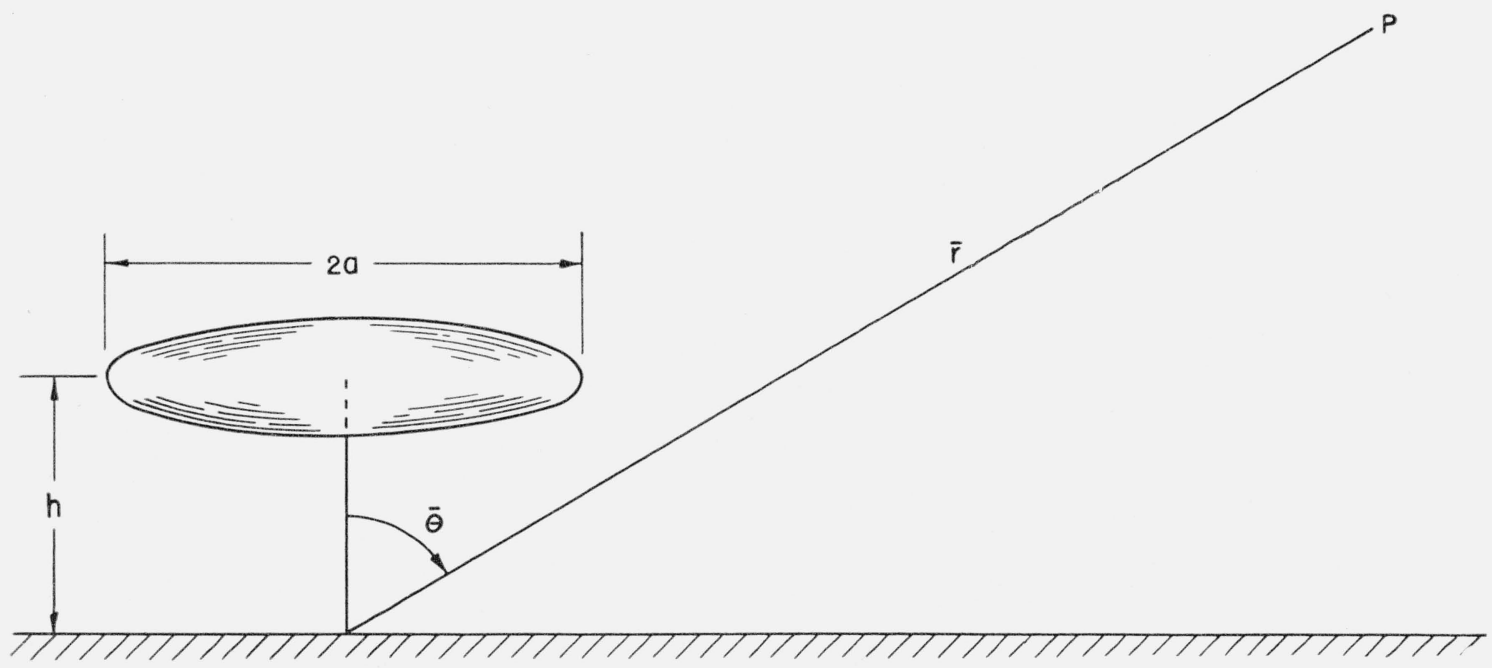

FIGURE 3. Coordinate system for describing radiation pattern. 
where terms in $(k a \sin \bar{\theta})^{3}$, etc. have been neglected. Noting that the radiation field for the vertical member in an $\bar{r}, \bar{\theta}$ coordinate system can be written

$$
H_{\phi}^{v}=\frac{i k I_{0} h e^{-i \beta \bar{r}}}{2 \pi \bar{r}} \sin \bar{\theta}
$$

it follows that the resultant radiation field is given simply by

$$
H_{\phi} \approx H_{\phi}^{v}\left(1-\frac{(k a)^{2} \cos ^{2} \bar{\theta}}{8}\right)
$$

neglecting terms of order $(k a)^{4}$.

\section{Conclusion}

The results indicate that the earth currents near a monopole antenna are not significantly modified by the presence of a symmetrical top loading. Therefore, in any calculation [4] of the energy absorbed in the ground system, it is usually sufficient to consider only the contribution from the currents in the vertical members (downleads). Furthermore, the radiation pattern of a loaded monopole at low radiofrequencies is essentially a dipole pattern being modified to small order by the currents in the top-loading structure. The effect of unsymmetrical top loading is considered in a sequel to this paper [5].

The author thanks Mrs. A. Murphy for carrying out the calculations and W. W. Brown, A. D. Watt, and H. A. Wheeler for informative discussions.

\section{References}

[1] Ronold W. King, Theory of linear antennas (Harvard Univ. Press, Cambridge, Mass., 1956).

[2] P. M. Morse and H. Feshbach, Methods of theoretical physics, Vol. II, p. 1326 (McGraw-Hill Book Co., New York, N.Y., 1953).

[3] C. E. Smith and E. M. Johnson, Performance of short antennas, Proc. IRE 35, 1026 (1947).

[4] J. R. Wait and W. A. Pope, Input resistance of l. f. unipole aerials with radiai wire earth systems, Wireless Engr. (London) 32, 131 (1955).

[5] H. L. Knudsen, Earth currents near a top-loaded monopole antenna with special regard to electrically small L- and T-antennas, J. Research NBS 62, 283 (1959) RP2961.

Boulder, Colo., January 27, 1959. 